\title{
Unterstützung des Managements von großen Netzen in der Logistik durch die systematische Bereitstellung von Methodenwissen
}

\author{
DiPL.-WIRT.-ING. STEPHAN KESSLER \\ DIPL.-WIRT.-MATH. BRITTA VON HAAREN \\ TECHNISCHE UNIVERSITÄT DORTMUND, SFB 559 „,MODELLIERUNG GROßER NETZE IN DER LOGISTIK“
}

\section{Zusammenfassung}

Das Management komplexer, interorganisatorischer und durch eine Vielzahl unterschiedlicher Beziehungen miteinander verknüpfter Strukturen und Prozesse, wie sie großen Netzen der Logistik zugrunde liegen, stellt für die verantwortlichen Akteure eine anspruchsvolle Aufgabe dar. Der nachfolgende Beitrag stellt einen systematischen Ansatz vor, mit dem Netzwerkmanager bei der Erfüllung dieser Aufgabe durch eine verbesserte Informationsgrundlage über verfügbare Methoden und Gestaltungsoptionen unterstützt werden sollen. Exemplarisch demonstriert wird die Funktionsweise dieses Ansatzes anhand des umfangreichen Instrumentariums des Netzwerk-Controlling, welches zur Schaffung von Transparenz in den unternehmensübergreifenden Prozessen zur Verfügung steht.

\section{Abstract}

The management considering complex structures and processes which are interlinked through a variety of different relations and organisational developments, as they underlie large networks in logistics, constitute a demanding assignment for responsible actors. The following contribution presents a systematic approach which supports network managers in the completion of this assignment by an improved fundament of information about available methods and constitutive options. The functionality of this approach is exemplified by means of extensive instruments of the network control which is available in company comprehensive processes in order to accomplish transparency.

\section{Einleitung}

Die Gestaltung und das Management großer Netze in der Logistik (GNL) erfordern den Einsatz von umfangreichem, interdisziplinärem Methodenwissen. Dieses Wissen wird von unterschiedlichen Akteuren im Rahmen praktischer Erfahrungen und wissenschaftlicher Aktivitäten erarbeitet und bereitgestellt. So beschäftigen sich die Wissenschaftler der Methoden- und Anwendungsprojekte des Sonderforschungsbereiches (SFB) 559 „Modellierung großer Netze in der Logistik“ aus Sicht der Ingenieurwissenschaften, der Informatik, der Betriebswirtschaftslehre, der Statistik und der Soziologie mit vielfältigen verschiedenen Fragestellungen im Kontext der Planung und des Betriebs logistischer Netzwerke. Hieraus ist ein umfangreicher Fundus an explizitem und implizitem methodischen Wissen zur Unterstützung der Planung und des Managements von GNL hervorgegangen. Um für Netzwerkmanager Nutzen stiftend und zielführend einsetzbar zu sein, muss dieses Wissen jedoch systematisch erfasst, strukturiert und dokumentiert und situationsspezifisch bereitgestellt werden. Der nachfolgende Beitrag stellt einen vom Teilprojekt M6 „Konstruktionsregelwerke“ entwickelten Ansatz vor, der die entsprechende Aufbereitung und Bereitstellung des Methodenwissens in einem internetbasierten Assistenzsystem - der so genannten Workbench - vorsieht, wodurch dem Netzwerkmanager eine verbesserte Informationsgrundlage hinsichtlich einsetzbarer Methoden und Gestaltungsoptionen zur Verfügung gestellt werden soll. Die Darstellung der Nutzung dieses Assistenzsystems erfolgt dabei anhand des im Rahmen des Teilprojekts M3 „Netzwerk-Controlling“ für GNL entwickelten Netzwerk-Controlling-Instrumentariums, mit dem der Netzwerkmanager Unterstützung bei der Analyse, Kontrolle und Verbesserung unternehmensübergreifender Prozessketten erfährt. 


\section{Workbench als Unterstützungssystem für die Gestaltung und das Management von GNL}

Der Prozess der Planung aber auch des Managements von GNL basiert auf der Interaktion zwischen Experten (Netzwerkplanern und -managern) und spezialisierten Assistenzsystemen, die durch die Bereitstellung spezifischer Informationen, Bewertungs- und Berechnungsgrundlagen Entscheidungsunterstützung für ganz bestimmte Aufgabenstellungen liefern. Darüber hinaus stehen den Experten zur Aneignung von Wissen über die Aufgabenstellung und zu ihrer Lösung einzusetzende Verfahren gedruckte oder elektronische Medien, themenspezifische Wissensmanagement-Anwendungen bzw. -Portale wie auch der eigene Erfahrungsschatz aus vorangegangenen Problemsituationen zur Verfügung. Während gedruckte und elektronische Medien den Nachteil haben, dass Wissen für eine bestimmte Aufgabenstellung aus sehr verteilten Quellen zusammengetragen werden muss, ist anzunehmen, dass Wissensmanagement-Anwendungen hier einen Vorsprung besitzen. Bisher verfügbare EDV-Systeme können aber die Praxis-Anforderungen nur unzureichend erfüllen: Sie legen ihren Fokus entweder eher auf Dokumentenverwaltungsfunktionen, wie Ablage und Suche, z.B.: Wissens-Portale, oder das erfasste Wissen wird nur relativ grob aufbereitet, z.B.: Best Practice- oder Projekt-Datenbanken. Oftmals basieren die enthaltenen Vernetzungen von Wissen auf automatischen Zuordnungen entsprechend einer sprachlichen Semantik oder ein Zugriff auf Wissen erfolgt vorrangig mit Hilfe einer Suchfunktion. Dies ist im Kontext konkreter Aufgabenstellungen beim Management von GNL zu unspezifisch.

\subsection{Zielsetzung des Assistenzsystems Workbench}

Zielsetzung des im Teilprojekt M6 entwickelten Assistenzsystems Workbench (vgl. auch [Laakmann02], [Laakmann05]) ist es daher, den Experten durch eine verbesserte Informationsgrundlage über die im Zuge der Planung oder des Betriebs eines GNL auftretenden Aufgabenstellungen zu unterstützen, z.B. indem konkret einsetzbare Methoden und Gestaltungsoptionen dargelegt werden (vgl. [Blutner06]). Dabei gilt es nicht, ggf. vorhandene Assistenzsysteme zu substituieren oder dem Experten Entscheidungen abzunehmen. Vielmehr erhält der Experte die Sicherheit, auf die geeigneten Systeme bzw. Methoden zurückgreifen zu können bzw. die richtigen Entscheidungen zu treffen.

Neben der Nutzung des in der Workbench enthaltenen Gestaltungswissens in einer konkreten Entscheidungssituation fällt es durch die verbesserte Verfügbarkeit von Gestaltungswissen in Form erfasster Wissensobjekte zu allen relevanten Aspekten leichter, sich einen Überblick über bestehendes Wissen in einem bestimmten Anwendungsgebiet der Logistikgestaltung zu verschaffen. Hierdurch wird für wissenschaftlich und konzeptionell orientierte Nutzergruppen eine Plattform geschaffen, die Ausgangspunkt für die Ableitung neuen Gestaltungswissens oder neuer Wissensobjekte ist. (vgl. [Wiesinger05])

\subsection{Aufbau und Strukturierung der Workbench}

Die zu Grunde gelegte Strukturierung der Workbench richtet sich am ganzheitlichen Gestaltungsansatz für Planungswissen großer Netze der Logistik [Laakmann05] und somit am Dortmunder Prozesskettenparadigma [Kuhn95] in Verbindung mit dem Vorgehensmodell zur prozessorientierten Logistikplanung [Kuhn07] aus. Diese theoretische Grundlage ermöglicht es, die Planung und das Management logistischer Systeme aus heterogenen Sichten heraus zu betrachten und eine Fülle von Gestaltungswissen zu ermitteln, welches in Form von Wissensobjekten frei geschnitten und dokumentiert werden kann. In der Workbench werden diese Wissensobjekte erfasst, vernetzt und strukturiert, um sie dem Netzwerkmanager im Kontext einer spezifischen Aufgabenstellung bereitstellen zu können. Abbildung 1 zeigt die dazu vorgesehenen grundlegenden Funktionen des Unterstützungssystems. Aus der Darstellung wird deutlich, dass bei der Workbench zwischen fünf funktionalen Bereichen (Hauptfunktionen) unterschieden wird, die in weitere Nebenfunktionen untergliedert sind:

- Erfassung: Eingabe und Pflege von Wissensobjekten

- Aufbereitung: Vernetzung und Aufbereitung von Wissensinhalten

- Recherche: Abfrage von Wissensinhalten

- Projektunterstützung: Unterstützung im Projektmanagement

- Administration: Administration und Benutzermanagement 


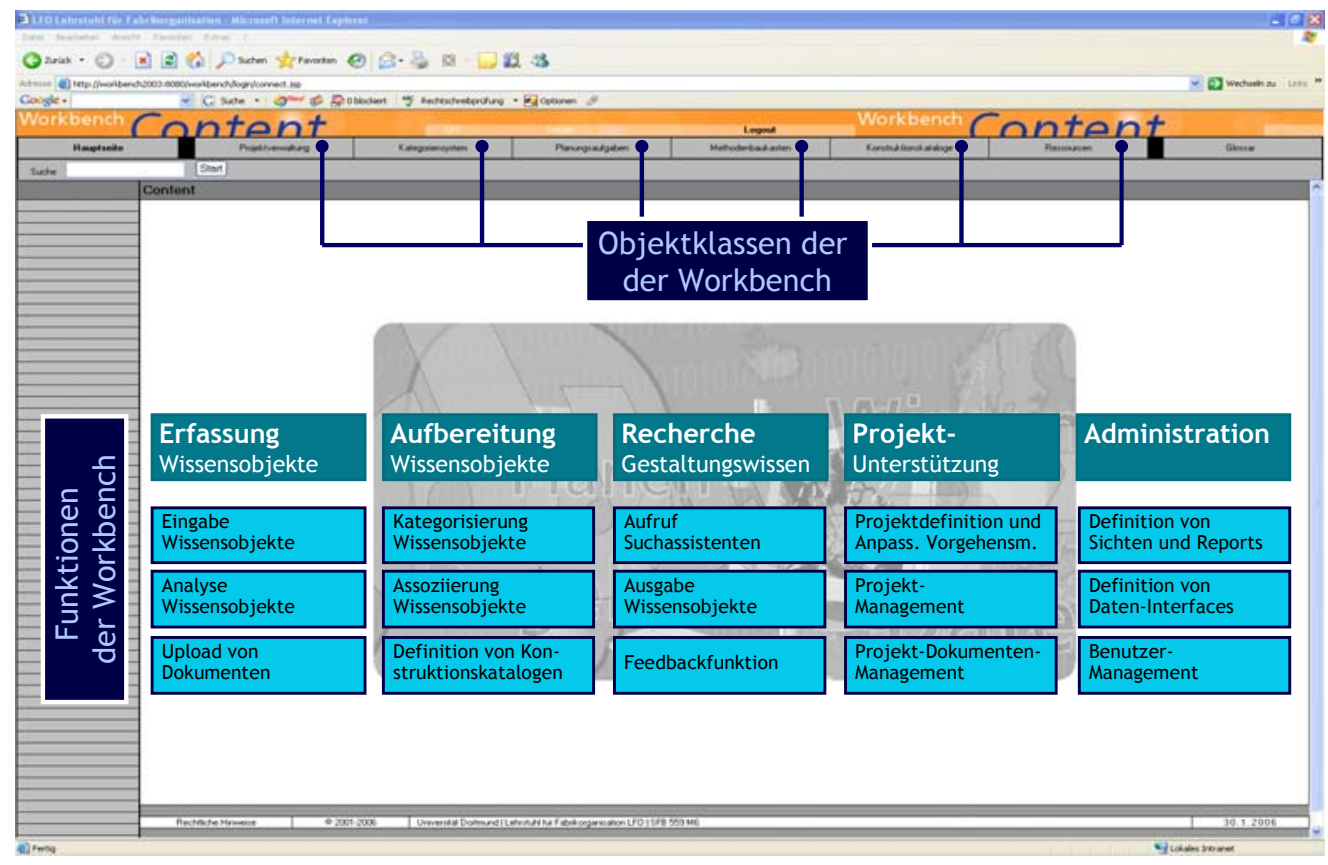

Abbildung 1: Funktionale Bereiche und Objektklassen der Workbench

Über die funktionale Gliederung hinaus, lassen sich die in der Workbench hinterlegten Wissensobjekte anhand inhaltlicher Kriterien in sechs verschiedene Objektklassen sowie ein zusätzliches Glossar einordnen (vgl. Abbildung 1, oben). Diesen Klassen können sämtliche in Bezug auf die Gestaltung und das Management logistischer Netzwerke relevanten Wissensobjekte zugeordnet werden. Den zentralen Ausgangspunkt bildet demnach der Planungsprozess, welcher in einer 3-Ebenen Gliederung (Aufgabe, Teilaufgabe und Schritt) bis auf elementare Aktivitäten dekomponiert werden kann (vgl. Abbildung 2, exemplarisch für die Planung von Güterverkehrszentren). Ein Planungsschritt als eine von i. d. R. mehreren elementaren, operativen Aktivitäten zur Durchführung einer übergeordneten Planungsaufgabe kann eine direkte Verknüpfung zu unterstützend einsetzbaren Methoden und zu betrachtenden Ressourcen aufweisen, die von den Wissensautoren der Workbench im Kontext als nützlich klassifiziert wurden. Methoden bzw. beanspruchte Ressourcen, welche im Zusammenhang mit der Gestaltung und dem Management logistischer Systeme genutzt bzw. eingesetzt werden, sind dazu - wie in der in diesem Artikel betrachteten Zusammenarbeit mit Teilprojekt M3 - dokumentiert, gesammelt und in eigenen Katalogen abgelegt worden.

Um neben der Zuordnung zu den einzelnen Objektklassen eine weitere, klassenübergreifende Klassifizierung der in der Workbench dokumentierten Wissensbausteine zuzulassen, wurde das Kategoriensystem definiert. Jedes Wissensobjekt (unabhängig davon, ob es als Planungsprozess, Methode oder Ressource klassifiziert wurde) lässt sich hiermit mit den Strukturelementen und Potenzialklassen des Prozesskettenelementes verknüpfen. 


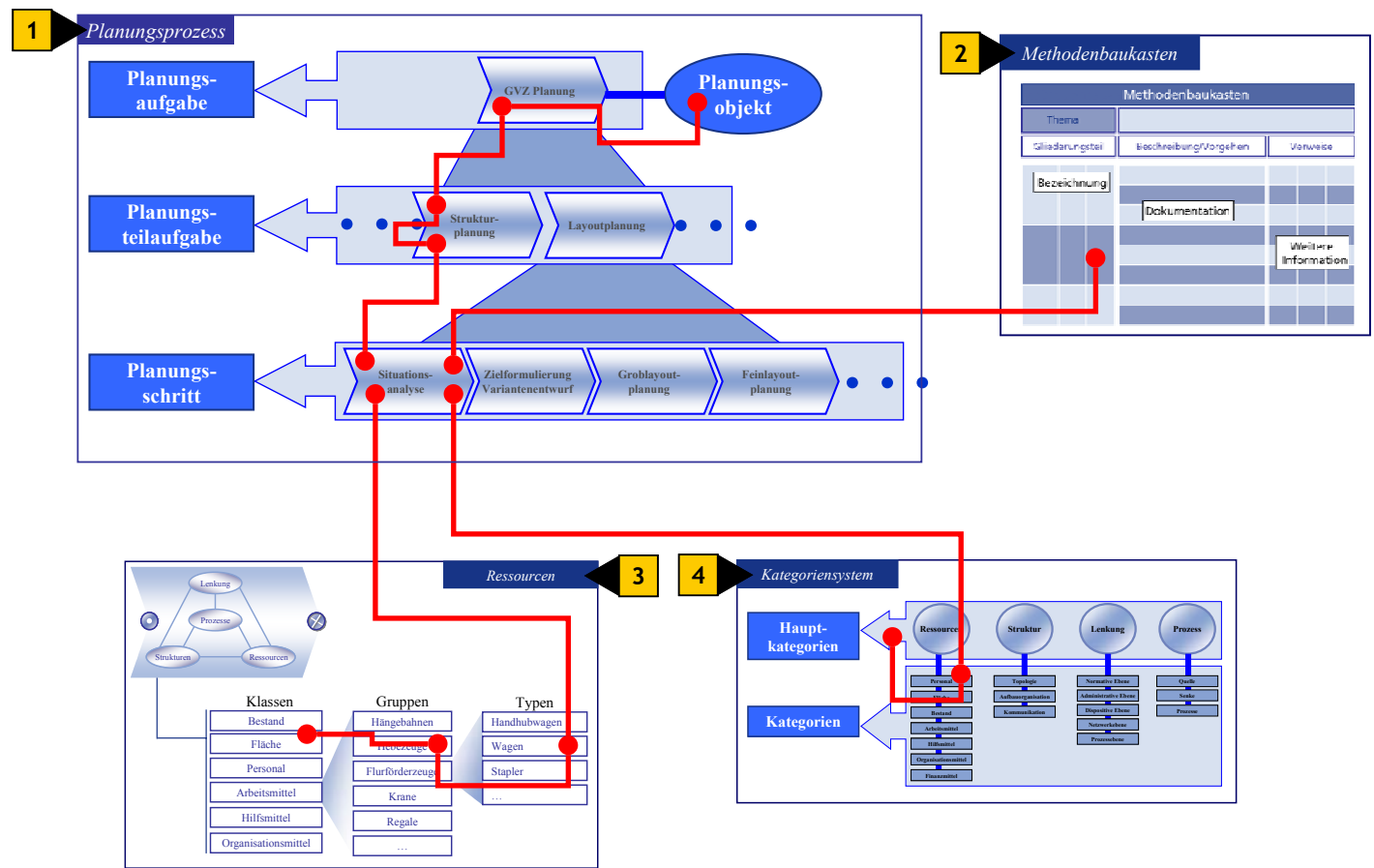

Abbildung 2: Vernetzung ausgewählter Objektklassen der Workbench

Weitere, in Abbildung 2 nicht dargestellte Objektklassen, sind die Projektverwaltung und die Konstruktionskataloge. In der Projektverwaltung werden ergänzend zu den allgemeingültigen Vorgehensmodellen und Gestaltungsparametern Beschreibungen bzw. Steckbriefe spezifischer Praxisprojekte als eigenständige Wissensobjekte abgelegt. Diese können im Sinne von Best-practice-Lösungen bei vergleichbaren Aufgabenstellungen im Zuge des Managements von GNL herangezogen werden [Kuhn99].

Die Objektklasse der Konstruktionskataloge dient letztendlich dazu, eine mehr-dimensionale Darstellung des hinterlegten Wissens zu ermöglichen und daraus übergeordnete Regeln abzuleiten. Die theoretisch-wissenschaftlichen Grundlagen zur Unterstützung des Gestaltungsprozesses (z.B. im Zusammenhang mit einer spezifischen Aufgabenstellung nutzbare allgemeingültige Vorgehensmodelle, einsetzbare Methoden und Ressourcen) auf der einen Seite werden dem dokumentierten Wissen zu erprobten und erfolgreich angewandten Lösungsansätzen aus der Praxis (die im Bereich der Projektverwaltung hintergelegt wurden) auf der anderen Seite gegenübergestellt. Aus dem resultierenden Abgleich können wertvolle Hinweise in Bezug auf die Konstruierbarkeit logistischer Systeme gewonnen werden.

Im Rahmen der Anwendung der Workbench ist es dem Netzwerkmanager damit möglich, sich dem abgelegten Wissen unter Ausnutzung der dargestellten Funktionen und Objektklassen auf verschiedenen Wegen zu „nähern“. Dabei wird die erste Auswahl und Spezifizierung der Aufgabenstellung dem Funktionsprinzip eines Konstruktionskataloges entsprechend immer vom Anwender vorgenommen. Im Folgenden wird das Vorgehen zur Ablage und Nutzung von Methodenwissen in der Workbench auszugsweise am Beispiel des Netzwerkcontrolling von großen Netzen in der Logistik demonstriert. Dazu wird zunächst kurz auf das Aufgabenspektrum dieses im Teilprojekt M3 behandelten Themenfeldes eingegangen, bevor die Integration der Ergebnisse dieses Teilprojektes in die Strukturen der Workbench thematisiert wird.

\section{Aufgabenspektrum des Netzwerk-Controlling}

Das Controlling eines Unternehmensnetzwerkes, insbesondere einer Supply Chain, ist Teil des Netzwerkmanagements und unterstützt dieses bei seinen Aufgaben. Das Hauptziel eines Netzwerk-Controlling (NWControlling) besteht darin, unternehmensübergreifende Prozesse zu analysieren, zu kontrollieren und wenn möglich zu optimieren (vgl. [Weber02], S. 52 ff). Die große Komplexität und Vielfalt an Aufgaben und Beziehungen erfordert dabei unterstützende Instrumente, die speziell für das Controlling von Netzwerken geeignet sind. Dabei reicht es nicht aus, Instrumente des unternehmensinternen Controlling unverändert auf Netzwerke zu übertragen; vielmehr müssen spezifische Methoden und Instrumente für Netzwerke entwickelt bzw. die bestehenden Möglichkeiten auf ihre Eignung für Netzwerke hin untersucht werden (vgl. [Darkow04], S. 113 f). Voraussetzung für ein netzwerkumfassendes Controllinginstrument ist die Schaffung von Transparenz über die gesamte Wertschöpfungskette hinweg (vgl. [Seuring01], S. 124). 
Das Zugreifen auf ein Planungsunterstützungssystem kann für einen Netzwerk-Controller sinnvoll und hilfreich sein und ihm bei seinem komplexen Aufgabenspektrum als gute Anleitung und instrumentelle Hilfe dienen. Auf diese Weise kann jeder Schritt im Controlling methodisch gesteuert und unterstützt werden.

\subsection{Teilaufgaben im Netzwerk-Controlling}

Die umfassende Aufgabe des NW-Controlling lässt sich in verschiedene Teilaufgaben zerlegen. Beispielhaft soll dies im Folgenden anhand eines Supply-Chain-Netzwerks dargestellt werden.

Als Gegenstand und Planungs- und Steuerungsobjekt steht bei allen Teilaufgaben der Geschäftsprozess in der Supply Chain (SC) im Mittepunkt. Ein SC-Geschäftsprozess lässt sich definieren als zusammenhängende, abgeschlossene Folge von einzelnen Aktivitäten zur Erstellung einer unternehmensübergreifenden Leistung (vgl. [Rosenkranz06], S. 3).

Die erste Teilaufgabe des SC-Controlling besteht darin, diejenigen Geschäftsprozesse zu bestimmen, welche die höchsten Verbesserungspotentiale bergen. Hierzu zählen z.B. Minimierungsmöglichkeiten hinsichtlich Kosten, Zeit oder Ressourcenverbrauch. Besonders gut eignet sich hierfür das Supply Chain-Benchmarking (BM), welches in verschiedenen Teilschritten Benchmarking-Objekte identifiziert, im Vergleich mit anderen Supply Chains (externes Benchmarking) oder ähnlichen Objekten innerhalb der eigenen Supply Chain (internes Benchmarking) analysiert und Optimierungsmöglichkeiten für einzelne Prozesse aufdeckt (vgl. [Böhnert99], S. 62 ff). Die einzelnen Phasen des SC-Benchmarking werden in Kapitel 3.2 genauer erläutert.

Als weitere Teilaufgabe des SC-Controlling sollen die Ist-Kosten der bestehenden Prozesse ermittelt werden. Hierfür eignet sich z.B. eine SC-Prozesskostenrechnung (PKR) bzw. eine Simulationsgestützte Prozesskostenrechnung, wie sie im Rahmen des Teilprojekts M3 „Netzwerk-Controlling“ des SFB 559 entwickelt worden ist (vgl. [Meinke06]). Der Vorteil gegenüber einer einfachen SC-PKR liegt bei der Simulationsgestützten PKR in der Unterstützung und der Bereitstellung technischer Kostentreiber aus der Simulation zur weiteren verursachungsgerechten Zuordnung der Kosten bzw. dem Aufzeigen der Auswirkungen von Änderungen an den Kostentreibern anhand der Simulation auf die Kosten.

Neben der Aufdeckung von Optimierungspotentialen und der Ermittlung der Ist-Kosten der SC-Geschäftsprozesse spielt die Festlegung von Zielgrößen, insbesondere Soll-Kosten, eine wichtige Rolle im SC-Controlling. Diese Aufgabe wird besonders gut durch ein SC-Target Costing erfüllt, welches sich zur Ermittlung von Zielpreisen und -kosten an den Anforderungen der Kunden und des Marktes orientiert (vgl. [Horváth93], S.3). Bei der Durchführung eines SC-Target Costing werden zuerst die aktuellen Marktbedingungen analysiert, um so einen möglichen Ziel-Verkaufswert zu erhalten, welchen Kunden noch bereit sind zu zahlen. Daraus ergeben sich zulässige Kosten, welche anschließend auf die einzelnen Wertschöpfungsstufen und weiter auf einzelne Prozesse, Komponenten, Teile und Funktionen aufgeteilt werden. Schließlich erfolgt eine Aufteilung dieser Plankosten nach einer festgelegten Gewichtung auf die Zulieferer (vgl. [Cooper99], S. 166).

Im Sinne des Controlling wird die Prozesskostenrechnung häufig als unterstützende Vorstufe zum Target Costing angesehen. Die durch die PKR ermittelten Ist-Kosten eines Prozesses werden für die weitere Prozessplanung unter Berücksichtigung der Zielkosten verwendet (vgl. [Mayer93], S. 77). Weiterhin müssen alle Teilaufgaben des NW-Controlling durch ein übergeordnetes Instrument gesteuert, überwacht und kontrolliert werden. Die ebenfalls im Rahmen des Teilprojektes M3 entwickelte NW-Balanced Scorecard als Steuerungsinstrument des NW-Controlling hat sich hierfür in der Praxis und Wissenschaft bereits mehrfach bewiesen (vgl. [Jehle06], [Zeller03]). In diese Scorecard lassen sich im Sinne des Controlling-Regelkreises die durch das SCBenchmarking, SC-Target Costing und die Simulationsgestützte PKR ermittelten Ziele, Kennzahlen und Benchmarks integrieren und anschließend mit Hilfe weiterer Methoden geeignete Maßnahmen auswählen und steuern (vgl. [Meinke06], S. 182 ff).

Wichtig zu erwähnen ist, dass das Netzwerk-Controlling keine abgeschlossene Aufgabe ist, sondern laufend durchgeführt werden muss, um aktuelle Veränderungen am Markt bzw. im Netzwerk aufzunehmen und immer wieder Verbesserungsmöglichkeiten auszuschöpfen (vgl. [Gruhn04], S. 231).

\subsection{Integration des Methodenwissen des NW-Controlling in die Work- bench}

Um das Controlling eines Netzwerks mit der in Kapitel 2 beschriebenen Workbench methodisch zu unterstützen, wird zunächst als Planungsaufgabe das Netzwerk-Controlling definiert und mit dem Planungsobjekt eines $S C$ - 
Geschäftsprozesses verbunden. Weiterhin wird die übergeordnete Aufgabe in die bereits genannten Planungsteilaufgaben SC-Benchmarking, SC-Prozesskostenrechnung, SC-Target-Costing und NW-Balanced Scorecard unterteilt. Jede dieser Teilaufgaben besteht aus mehreren Phasen bzw. einzelnen Planungsschritten. Der Methodenbaukasten der Workbench liefert eine umfangreiche Sammlung verschiedener Methoden und Instrumente, die zur Unterstützung der einzelnen Schritte ausgewählt werden können. Dieser Vorgang soll im Folgenden beispielhaft an der Planungsaufgabe des SC-Benchmarking, also der Identifikation zu verbessernder Prozesse, erläutert werden.

Abbildung 3 zeigt die formale Einordnung der übergeordneten Planungsaufgabe des Netzwerk-Controlling, der aufgeteilten Planungsaufgaben und auszugsweise einzelner Planungsschritte.

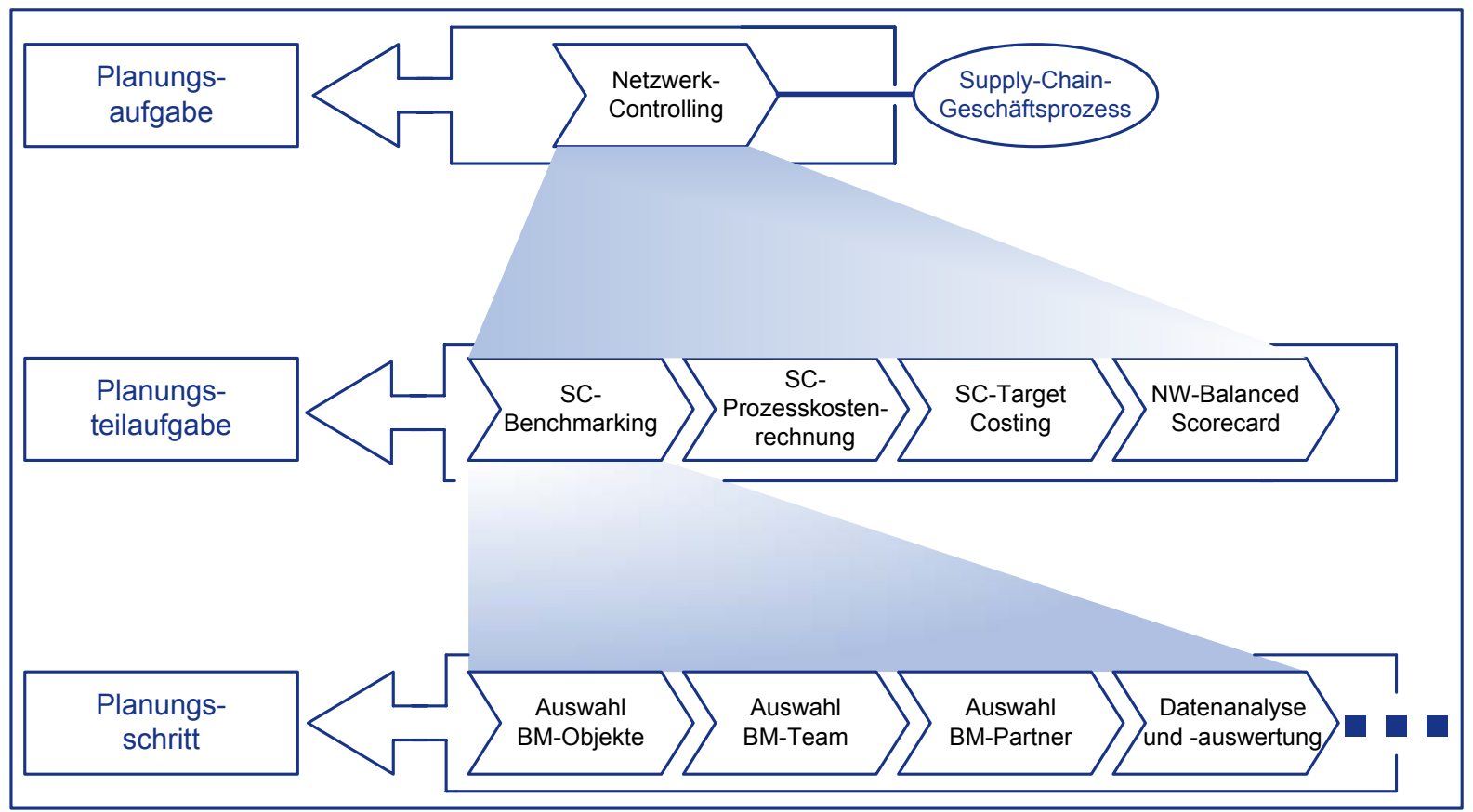

Abbildung 3: Planungsaufgabe Netzwerk-Controlling

Abbildung 4 veranschaulicht noch einmal detaillierter die einzelnen Phasen im Benchmarking-Prozess (entnommen aus ([Meinke06], S. 174). Zur Unterstützung dieser Planungsschritte können aus dem Methodenbaukasten der Workbench verschiedene geeignete Instrumente und Basis-Methoden ausgewählt werden. So kann bspw. bei der Auswahl der Benchmarking-Objekte und Benchmarking-Partner eine ABC-Analyse oder ein Bewertungssystem hilfreich sein. Zur Bestimmung des BM-Teams könnte ein auf Erfahrungen mit den Mitarbeiten beruhendes formalisiertes Brainstorming vorgenommen werden, wie es als Methodenvorschlag ebenfalls in der Workbench zur Auswahl steht. Dagegen kann die Datenanalyse und -auswertung auf Basis einer Effizienzanalyse geschehen. Die Maßnahmenauswahl schließlich kann durch die Basismethode einer Nutzwertanalyse, also eines Scoring-Modells erfolgen. 


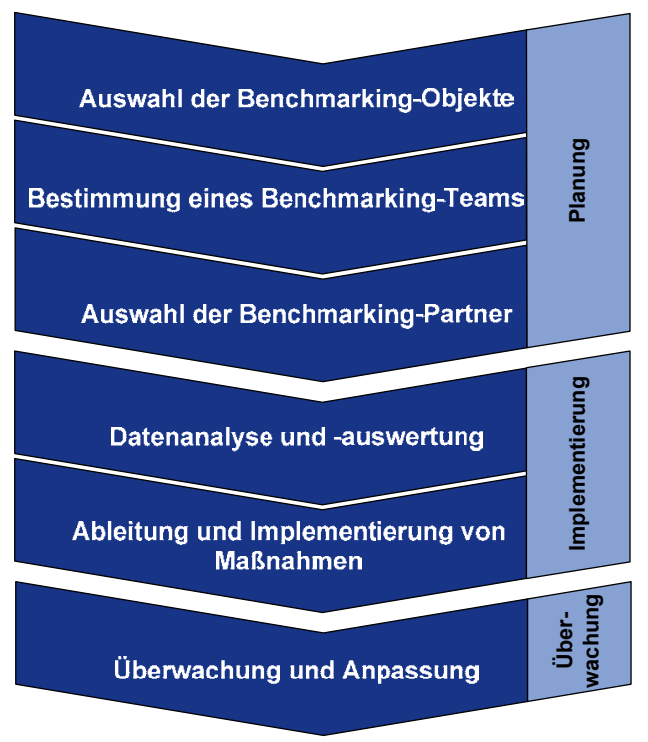

Abbildung 4: Phasen des Supply Chain-Benchmarking

Dabei ist die Auswahl dieser Instrumente variabel und an die jeweiligen Bedürfnisse und das evtl. bereits vorhandene und bestehende Methodenspektrum im Netzwerk anpassbar. Eine mögliche Auswahl bildet Abbildung 5 ab.

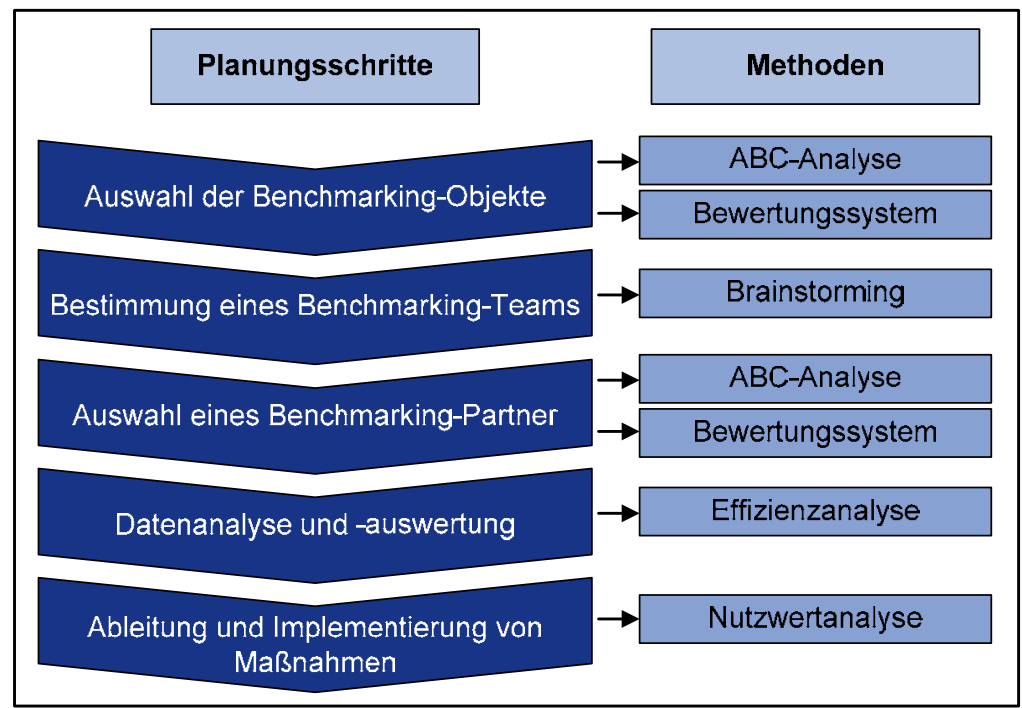

Abbildung 5: Basismethoden zur Unterstützung der Planungsschritte des SC-Benchmarking

Ähnlich wie beim SC-Benchmarking lassen sich auch bei den anderen Planungsteilaufgaben wie z.B. beim SCTarget Costing aus dem Methodenbaukasten der Workbench verschiedene Basismethoden auswählen, um die einzelnen Planungsschritte zu instrumentalisieren. Die Auswahl ist dabei eher subjektiv und vom Management des Netzwerk-Controlling abhängig. Die Workbench stellt alle Methoden und das gesammelte Methodenwissen aus vielen verschiedenen Bereichen und Planungsaufgaben eines Managements zur Verfügung. Die mögliche Eignung der einzelnen Methoden für die vorliegende Aufgabe geht dabei aus den hinterlegten Methodenbeschreibungen hervor und muss passend ausgewählt werden. Abbildung 6 gibt einen Ausschnitt aus der Workbench wieder, welcher die Planungsteilaufgabe des SC-Benchmarking abbildet. 


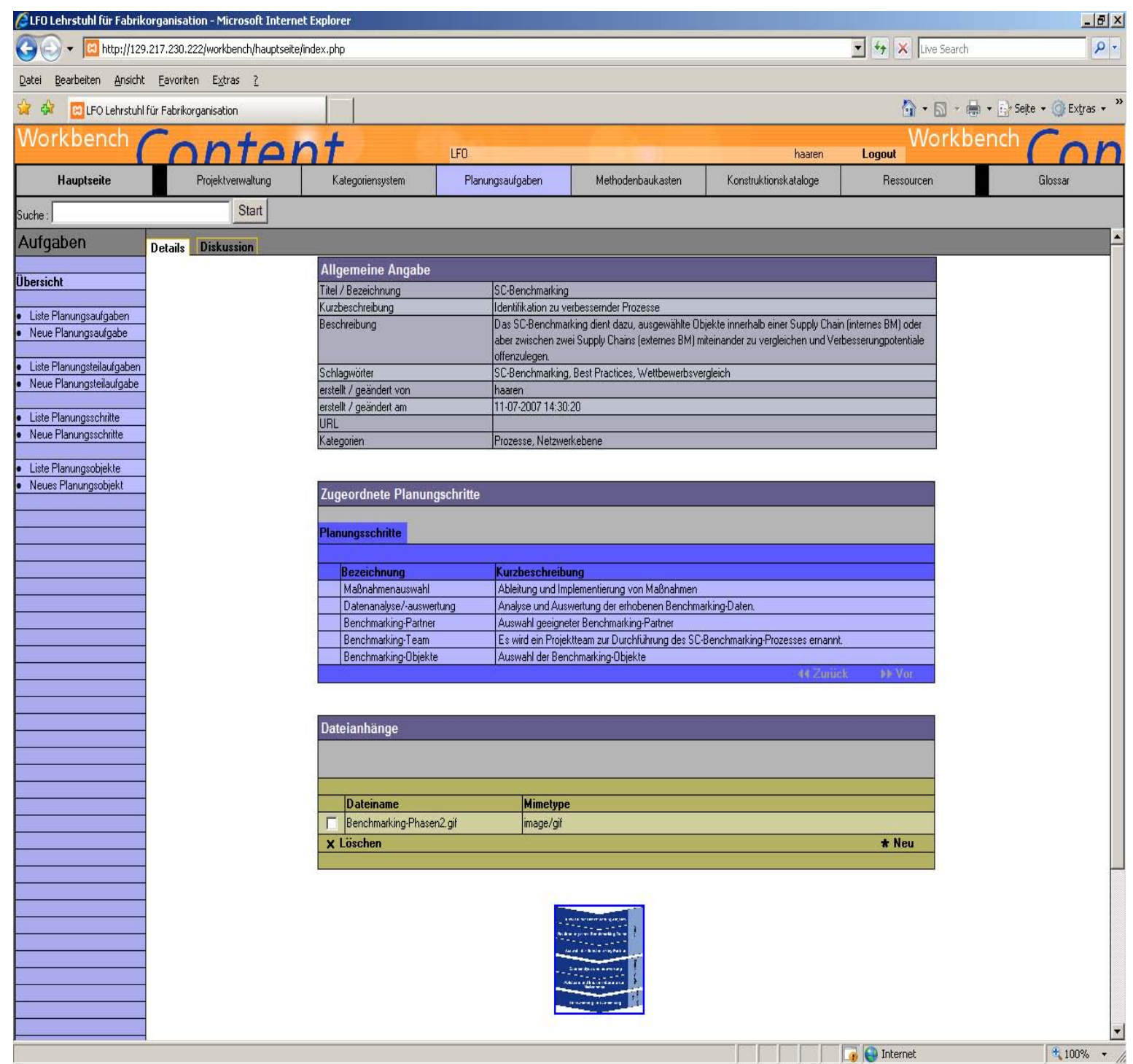

Abbildung 6: Beispiel einer Planungsteilaufgabe in der Workbench

\section{Fazit}

Im vorliegenden Bericht wurde beispielhaft anhand der Planungsaufgabe des Netzwerkcontrolling die Vorteilhaftigkeit der systematischen Erfassung von Methodenwissen in der Workbench aufgezeigt. Mit Hilfe der hinterlegten Planungsteilaufgaben und Instrumente wird der Anwender schrittweise bei der Ausführung seiner Aufgaben unterstützt und kann aufgrund der Vielzahl der erfassten Methoden sowie ihrer detaillierten Beschreibungen die für ihn geeignete Methode auswählen.

Dabei bleibt das Methodenwissen in der Workbench nicht auf einzelne betriebswirtschaftliche Bereiche, wie das Netzwerkcontrolling, begrenzt, sondern ermöglicht die Erfassung vielseitiger Aufgaben aus allen Bereichen eines Netzwerks oder Unternehmens. So haben bspw. neben dem Teilprojekt M3 bereits das Teilprojekt A2 "Beschaffungsketten" und Teilprojekt A4 "Netze und Güterverkehrszentren" weitere Planungsaufgaben und Methoden in die Workbench aufgenommen.

Im Sinne der Vollständigkeit und Weiterentwicklung lässt sich die Workbench stetig um weitere Objekte, Aufgaben und Methoden ergänzen, um ein möglichst großes Spektrum für die Anwender zur Verfügung zu stellen. 


\section{Literatur}

[Blutner06] Blutner, D.; Cramer, S.; Krause, S.; Nagel, L.; Reinholz, A.; Witthaut, M.: Ergebnisbericht der Arbeitsgruppe 5 "Assistenzsysteme für die Entscheidungsunterstützung". Technical Report - Sonderforschungsbereich 559 "Modellierung großer Netze in der Logistik" 06009, 2006 https://eldorado.unidortmund.de/bitstream/2003/24373/1/Technical\%20Report\%2006009.pdf

[Böhnert99] Böhnert, A.-A.: Benchmarking. Charakteristik eines aktuellen Managementinstruments. Hamburg: Dr. Kovač, 1999.

[Cooper99] Cooper, R.; Slagmulder, R.: Supply Chain Development for the Lean Enterprise: Interorganizational Cost Management. Portland: Productivity Press, 1999.

[Darkow04] Darkow, I.-L.; Richter, M.: Supply Chain Controlling. In: Baumgarten, H.; Darkow, I.-L.; Zadek, H.: Supply-Chain-Steuerung und Services. Logistik-Dienstleister managen globale Netzwerke - best practices, Berlin: Springer, 2004, S. 113-122.

[Gruhn04] Gruhn, V.; Koch, G.: Mit Prozess-Benchmarking zu besseren Ideen. Ein Führungsinstrument zur Motivation und Leistungssteigerung. In: Versicherungswirtschaft, 59(2004)4, S. 231-234.

[Horváth93] Horváth, P.; Niemand, S.; Wolbold, M.: Target Costing - State of the Art. In: Horváth, Peter: Target Costing. Marktorientierte Zielkosten in der deutschen Praxis. Stuttgart: Schäffer-Poeschel, 1993, S. 1-27.

[Jehle02] Jehle, E.; Stüllenberg, F.; Schulze im Hove, A.: Die Netzwerk-Balanced Scorecard als Instrument des Supply Chain Controlling. In: Supply Chain Management, 2(2002)4, S. 19-25.

[Kuhn95] Kuhn, A.: Prozessketten in der Logistik. Entwicklungstrends und Umsetzungsstrategien. Dortmund: Verlag Praxiswissen, 1995

[Kuhn99] Kuhn, A.: Prozesskettenmanagement, Erfolgsbeispiele aus der Praxis. Dortmund: Verl. Praxiswissen, 1999.

[Kuhn07] Kuhn, A.; Schmidt, A.; Beller, M.: Neue Vorgehensmodelle zur prozessorientierten Logistikplanung. Jahrbuch der Logistik 2007. Handelsblatt Fachblatt, Düsseldorf 2007

[Laakmann02] Laakmann, F. et al.: Workbench - Workbench zur Unterstützung der Planung logistischer Netze. Technical Report - Sonderforschungsbereich 559 ,Modellierung großer Netze in der Logistik“ 02003, Dortmund, 2002 https://eldorado.unidortmund.de/bitstream/2003/5483/1/02003.pdf

[Laakmann05] Laakmann, F.: Konstruktionsmethodischer Gestaltungsansatz für die Logistik. Umsetzung eines Modellierungskonzeptes für Planungswissen in der Logistik, Dissertation Universität Dortmund, Dortmund 2005.

[Mayer93] Mayer, R.: Target Costing und Prozesskostenrechnung. In: Horváth, P.: Target Costing. Marktorientierte Zielkosten in der deutschen Praxis. Stuttgart: Schäffer-Poeschel, 1993, S. 75-92.

[Meinke06] Meinke, A.: Cost-oriented Supply Chain Management and Supply Chain Controlling Configuration and Combination of Instruments, Dissertation, Universität Dortmund, 2006.

[Rosenkranz06] Rosenkranz, F.: Geschäftsprozesse. Modell- und computergestützte Planung. Berlin: Springer, 2006.

[Seuring01] Seuring, S.: Supply Chain Costing. Kostenmanagement in der Wertschöpfungskette mit Target Costing und Prozesskostenrechnung. München: Vahlen, 2001.

[Weber02] Weber, J.; Bacher, A.; Groll, M.: Instrumente des Supply Chain Controlling. In: Bundervereinigung Logistik: Wissenschaftssymposium Logistik der BVL, München, BVL, 2002, S. 85-97.

[Wiesinger05] Wiesinger, G.: Workbench: A Tool for Reconfigurable and Agile Factory and Supply Chain design. Vortrag, 1st International Conference on Changeable, Agile, Reconfigurable and Virtual Production (CARV 2005), (Organization: Institute for Machine Tools and Industrial Management (iwb)), Technische Universität München, Garching, 22.-23. September, 2005.

[Zeller03] Zeller, A.J.: Controlling von Unternehmensnetzwerken: Bestandsaufnahme und Lückenanalyse. FORWIN-Bericht, Bamberg u.a., Bayrischer Forschungsverbund Wirtschaftsinformatik, 2003. 